\title{
Optimization of Positron-Lifetime Measurement Geometry Based on Geant4 Simulation
}

\author{
M. Saro*, M. Petriska And V. Slugeñ \\ Institute of Nuclear and Physical Engineering, Faculty of Electrical Engineering and Information Technology, \\ Slovak University of Technology, Ilkovičova 3, 81219 Bratislava, Slovakia

\begin{abstract}
Proper choice of measuring geometry and experimental setup of nuclear instrumentation modules and photomultipliers is a key element which affects substantial positron lifetime measurement properties: count rate and time resolution. An adequate compromise must be found, when it comes to geometry of measurement. The optimal geometry using three detector layout is inspected in this paper. During our work, we concentrated on the simulation of XP2020Q photomultipliers and the $\mathrm{BaF}_{2}$ scintillator material. The Geant4 simulation allows to estimate an influence of the measuring geometry on detection efficiency and to choose the most appropriate crystals dimensions and positions. As mentioned in paper of Bečvaŕ et al., slight changes in geometry result in distortion or improvement of measured results. Experimental results already showed, changes of start crystals dimensions can result in significant increase in count rate of three-detector measurement.
\end{abstract}

DOI: 10.12693/APhysPolA.132.1634

PACS/topics: 29.30.Ep, 29.30.Kv

\section{Introduction}

Positron annihilation spectroscopy is a traditional technique used in materials science. This technique allows non-destructive testing of electronic structure of materials, mechanical and radiation-induced defects [1]. ${ }^{22} \mathrm{Na}$ radioactive isotope is frequently used in conventional positron lifetime measurements as a positron source. The emission of positrons is accompanied by a 1.274 MeV photon.

Positrons, emitted by the source, enter studied material sample, where are slowed down to thermal energies and eventually annihilate, emitting a pair of annihilation photons with energies of $0.511 \mathrm{MeV}$. Time between birth of a nuclear photon and birth of annihilation photons is positron lifetime. For detection of these photons, fast scintillation detectors are used, where positron lifetime is measured as time delay between detection of nuclear photon and detection of two annihilation photons.

Conventional and digital method of information acquirement from detectors uses same principle: fast signals related to moments of emission of corresponding photons are generated by constant-fraction differential discriminators (CFDDs). These signals are lead to the start and stop inputs of a time-to-amplitude converter (TAC). Coarse energy selection is provided by the CFDDs, too. The fast coincidence unit (FCU) produces gate signal for the TAC provided that the coincidence event of photons with proper energies occurred. Amplitude of the TAC output signal, which is proportional to time delay $t$ between the birth and the annihilation photons, is digitalized by an analog-to-digital converter (ADC) and the

*corresponding author; e-mail: matus.saro@stuba.sk event is then stored at corresponding address of a histogramming memory $[1,2]$.

In this process, there are several factors, which can influence a positron annihilation lifetime spectrum. The first one is the propagation of nuclear and annihilation photons from the point of birth to the point (or points) of interaction in a detector. The propagation time is a random value and depends on the size of detectors, on the distance between them, on the number of the photon scattering in the detectors and environment. The second is the process of light collection in the scintillation crystal. The third is the process of pulse formation in the photomultiplier tube. The forth is the instability of thresholds in CFDD [2,3].

In order to decrease influence of first mentioned factor in three detector layout, Monte Carlo simulation was made. In three detectors layout of positron annihilation lifetime spectroscopy (PALS), two opposite detectors (stop detectors) detect annihilation photons and one detector, placed perpendicularly to stop detectors connecting line, detects nuclear photons created by positron emission.

Distortion of measurement data can be caused by detecting scattered photons, which were originally emitted in different direction. In this work we call this kind of event "false" detected event. Other distortion occurs when more photons are detected at the time which causes possible pile-up. Suppressing of these events can be done by detecting thresholds or proper geometry choice. To choose optimal arrangement we were observing efficiency of three detectors layout PALS system detection.

\section{Simulation}

The simulation of PALS spectrometer described is based on GEANT4 toolkit. It is capable of modeling transport, interaction and processes created by photons 
and beta particles emitted from the source and other secondary particles from interactions $[3,4]$.

Modeled setup includes four different layouts. All four cases include two $\mathrm{BaF}_{2}$ scintillators with a diameter of $25.4 \mathrm{~mm}$ and height $12 \mathrm{~mm}$ as stop detectors, $4 \mathrm{~cm}$ distant from the center of coordination system. Between them, Si sample is placed with height $1 \mathrm{~cm}$, width $1 \mathrm{~cm}$ and depth $2 \mathrm{~mm}$. Point source is placed in the center of coordination system. The first layout includes the same cylindrical scintillator as stop detectors $4 \mathrm{~cm}$ distant from the sample. The second layout includes $4 \mathrm{~mm}$ lead plate in front of the start detector. In the third layout a cone detector with diameter at the base $36 \mathrm{~mm}$, diameter at the top $25.4 \mathrm{~mm}$ and height $16 \mathrm{~mm}$, was used as start detector. The fourth layout includes lead plate in front of cone start detector (see Fig. 1).

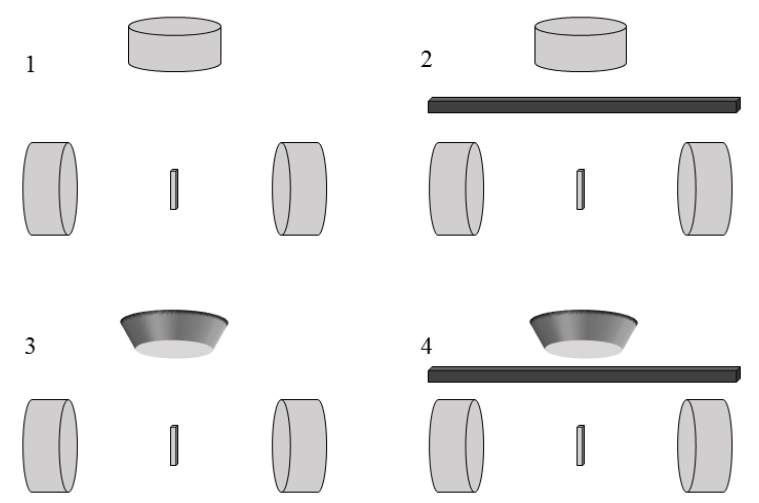

Fig. 1. Geometry of layouts.

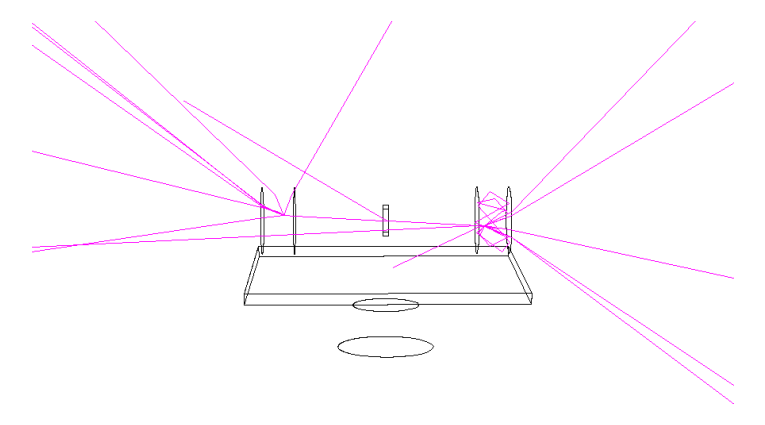

Fig. 2. One simulation event for layout 4 .

For each arrangement of simulated layout 1 million of source decays are generated.

Then two annihilation photons are emitted as a result of the positron annihilation. Trajectory step method is applied [4]. A step corresponds to the trajectory of a particle between discrete interactions. In our GEANT4 model we involved photon interactions and charged particle interactions. From Geant4 class G4SteppingAction, which is class called to access state of particle during its movement [5], we recall information about the particle using track method.
For our purposes, we track particles from source decays. If these particles reach their correspondent detector, where they cause scintillation process, this event is saved as hit at the detector. Additionally, scattered photons are tracked as well, using same principle as by "true events" (see Fig. 2).

\section{Results and discussion}

Simulations were carried out for four different layouts (see Fig. 1). Every layout under our study showed differences in the efficiency of detection.

Detection efficiencies in different layouts.

TABLE I

\begin{tabular}{c|c|c|c}
\hline \hline Layout & True events & True \& false events & Start detector \\
\hline 1 & $3.759 \mathrm{E}-07$ & $1.115 \mathrm{E}-06$ & $3.050 \mathrm{E}-02$ \\
\hline 2 & $3.070 \mathrm{E}-07$ & $6.666 \mathrm{E}-07$ & $2.950 \mathrm{E}-02$ \\
\hline 3 & $2.309 \mathrm{E}-07$ & $1.063 \mathrm{E}-06$ & $1.866 \mathrm{E}-02$ \\
\hline 4 & $3.057 \mathrm{E}-07$ & $1.026 \mathrm{E}-06$ & $1.683 \mathrm{E}-02$
\end{tabular}

As can be seen in Table I, in general there is a lower detection efficiency in layouts which include lead plate. However, this efficiency is strongly influenced by scattered photons, which cause scintillation signal in start detector. The cylindrical scintillator has in general $40 \%$ higher detection efficiency than cone scintillator. Our assumptions on this finding are that cylindrical scintillator covers bigger spatial angle. When start detector is placed behind lead plate, number of events which cause detection is $62 \%$ smaller. This means that ratio of true and false events increases, when using lead plate. As stated before, this effect can be partially suppressed by thresholding, but this method does not reduce false event number from pile-up.

The results state that better ratio of true/false coincidences can be increased by placing lead plate in front of start detector. However, this can result in higher number of backscattered photons into stop detectors, which are supposed to have energy threshold slightly below $511 \mathrm{keV}$, to suppress detecting of backscattered photons with certain energy loss. Reducing the probability of detection annihilation photons from two different events at stop detectors is in this case provided by having two stop detectors. We find layout no. 2 (cylindrical start detector with lead plate) as best solution out of four proposed layouts (see Fig. 1), when distance between sample with source and detectors has to be kept.

\section{Conclusion}

Three detector PALS layout was inspected in terms of detection efficiency. As shown, the efficiency from true coincidences was highest when using cylindrical scintillator behind lead plate. Placing of lead plate in front of start detector causes $62 \%$ percent increase in true event detection and therefore smaller distortion in lifetime spectrum. 


\section{Acknowledgments}

This article was created with the support of VEGA $1 / 0339 / 16$ and $1 / 0104 / 17$.

\section{References}

[1] F. Bečvář, J. Čížek, L. Lešták, I. Novotný, I. Procházka, F. Šebesta, Nucl. Instrum. Methods Phys. Res. A 443, 557 (2000).

[2] R. Krause-Rehberg, H.S. Leipner, Positron Annihilation in Solids. Defect Studies, Springer, Berlin 1999, p. 378 .
[3] L.Yu. Dubov, V.I. Grafutin, Yu.V. Funtikov, Yu.V. Shtotsky, L.V. Elnikova, Nucl. Instrum. Methods Phys. Res. B 334, 81 (2014).

[4] S. Agostinelli, J. Allison, K. Amako, J. Apostolakis, H. Araujo, P. Arce, M. Asai, D. Axen, S. Banerjee, G. Barrand, et al., Nucl. Instrum. Methods Phys. Res. A 506, 250 (2003).

[5] Geant4 Collaboration, Geant4 Application Developers Guide. 